\title{
Demixing behavior in two-dimensional mixtures of anisotropic hard bodies
}

\author{
Yuri Martínez-Ratón* \\ Grupo Interdisciplinar de Sistemas Complejos (GISC), Departamento de Matemáticas, \\ Escuela Politécnica Superior, Universidad Carlos III de Madrid, \\ Avenida de la Universidad 30, E-28911 Leganés, Madrid, Spain. \\ Enrique Velascd \\ Departamento de Física Teórica de la Materia Condensada and Instituto de Ciencia de Materiales Nicolás Cabrera, \\ Universidad Autónoma de Madrid, E-28049 Madrid, Spain. \\ Luis Medero那 \\ Instituto de Ciencia de Materiales, Consejo Superior de Investigaciones Cientificas, E-28049 Cantoblanco, Madrid, Spain.
} (Dated: February 2, 2008)

\begin{abstract}
Scaled particle theory for a binary mixture of hard discorectangles and for a binary mixture of hard rectangles is used to predict possible liquid-crystal demixing scenarios in two dimensions. Through a bifurcation analysis from the isotropic phase, it is shown that isotropic-nematic demixing is possible in two-dimensional liquid-crystal mixtures composed of hard convex bodies. This bifurcation analysis is tested against exact calculations of the phase diagrams in the framework of the restricted-orientation two-dimensional model (Zwanzig model). Phase diagrams of a binary mixture of hard discorectangles are calculated through the parametrization of the orientational distribution functions. The results show not only isotropic-nematic, but also nematic-nematic demixing ending in a critical point, as well as an isotropic-nematic-nematic triple point for a mixture of hard disks and hard discorectangles.
\end{abstract}

PACS numbers: $64.70 . \mathrm{Md}, 64.75 .+\mathrm{g}, 61.20 . \mathrm{Gy}$

\section{INTRODUCTION}

The demixing behavior of hard-core three-dimensional additive mixtures composed of particles without orientational degrees of freedom, in particular the hard-sphere and parallel hard-cube systems, has been recently studied in depth by several authors using Monte Carlo simulation techniques 1, 2, 3] and theory [4, 5]. The main conclusions that can be drawn from these studies is that fluid-fluid demixing is always metastable with respect to fluid-solid demixing for asymmetric mixtures in which the solid phase is composed of big particles and the fluid phase is enriched in small particles (demixing behavior similar to the one found in mixtures of parallel hard cubes on a cubic lattice [6]). The physics behind this demixing behavior is known as the depletion effect [], and can be explained as the effective attraction between two large particles due to the uncompensated osmotic pressure exerted by the small particles when the two excluded volumes between big and small particles overlap. Thus in the demixed phases enriched by each of the species the accessible volumes to the particles is maximized and as a consequence the total configurational entropy is increased.

Three-dimensional mixtures of additive anisotropic particles possess a demixing behavior which differs from

\footnotetext{
*Electronic address: yuri@math.uc3m.es

${ }^{\dagger}$ Electronic address: enrique.velasco@uam.es

‡Electronic address: 1.mederos@icmm.csic.es
}

that of mixtures composed of isotropic particles because of the presence of particle orientational degrees of freedom. It has been shown that demixing strongly depends on the shape of the particles (spherical, oblate or prolate) 8, 9, 10, 11, 12, 13, 14] and for a particular geometry, on its aspect ratio (the ratio between the characteristic lengths of particles) [15, 16, 17]. For example, one of the demixed phases can be an oriented phase, i.e., a nematic $(\mathrm{N})$ phase where the particles are aligned on average along the nematic director. It is easy to predict the most common demixing scenario, it will proceed between a $\mathrm{N}$ phase composed of the longer particles and an isotropic (I) phase composed of the short-particle component. In this case the excluded volume between the large particles is minimized by their parallel alignment. I-I and $\mathrm{N}-\mathrm{N}$ demixing was also found in mixtures of anisotropic particles 8, 9, 10, 11, 12, 13, 14, 15, 16, 17], showing that fluid-fluid demixing is a common scenario in these mixtures, which has recently been confirmed experimentally [18].

The scaled particle theory (SPT) was initially developed for hard spheres [19 and later extended to anisotropic particles [20, 21, 22]. The usual formulation of SPT for mixtures of hard convex bodies restricted to the isotropic orientational phase has as a main ingredient the expression for the second virial coefficients as a function of the volumes, surface areas, and mean curvatures of hard convex bodies [23, 24]. This exact result obtained in the 1950's 23] was used to show that in two dimensions I-I demixing is not allowed 25], the gain in accessible volume after demixing is much lower in two 
dimensions, which explains the stability of mixtures with respect to I-I demixing at any composition. This result has been confirmed by other theories constructed from the expression of the direct correlation function in terms of the geometric measures of the particles [10], in the same spirit as the fundamental measure theory (FMT) for hard spheres [26, 27]. Some authors have studied the possibility of demixing in a mixture of perfectly oriented two-dimensional additive particles 10, arriving at the same conclusion, the mixture is always stable. However, in contrast to these findings, we will show in section III that the SPT approximation does predict N-N demixing in the limit of parallel alignment. Also, the previous negative results cannot be taken as definite, since the suppression of orientational fluctuations is a crude approximation to study the possibility of $\mathrm{N}-\mathrm{N}$ demixing, so that the question about the existence of demixing (I-N or N-N) in two dimensions is still open. Note that we explicitly distinguish I-N demixing from the usual orientational I-N phase transition. The reason for this will be explained later in Sec. IV C.

As opposed to additive mixtures, nonadditive mixtures of hard disks can demix, as was shown by several authors [28, 29]. This result is not surprising since the demixed phases, composed of practically the same kind of species, are approximately additive and, as a consequence, have less excluded volumes between any pair of particles compared to the mixed state. Nonadditive mixtures made up of anisotropic particles have not been analyzed so far. The coupling between nonadditiveness in the interactions and orientational degrees of freedom is expected to give rise to interesting phenomenology, which is worth investigating. We will not pursue these aspects in the present paper.

The purpose of this work is to shed light on the question about the existence of I-N or N-N demixing in twodimensional mixtures of additive hard anisotropic particles. We have used the SPT formalism specified for a mixture of hard rectangles (HR) and hard discorectangles (HDR), and applied a bifurcation analysis from the isotropic phase to find the possibility of I-N demixing. This analysis has been shown to be useful in the study of phase transitions with symmetry breaking. In particular it was used to study the I-N transition in two dimensions in a one-component system of hard needles 30], and in a system composed of self-assembled rods 31] in the same two-dimensional Onsager limit. Using the bifurcation analysis, we have found that, even for a system of HDR (where the I-N transition is continuous in the one-component limit, as indicated by simulation 32 and density-functional theory 33]) the mixture can demix in two phases of different composition. In addition, we have calculated the phase diagrams of different mixtures of HDR and confirmed this demixing scenario. In some mixtures we have also found a N-N phase separation which ends in a critical point, as well as a triple coexistence between an I phase and two different nematics.
The paper is organized as follows. In Sec. II we present the theoretical model, and in Sec. III the bifurcation analysis. The results from this analysis are shown for HDR in Sec. IV A, for HR in Sec. IV B, and a check using a very simple model (Zwanzig approximation) is presented in Sec. IV C. Section V shows the results from the calculations of the phase diagrams of HDR mixtures. Finally some conclusions are drawn in Sec. VI.

\section{MODEL}

The key quantity in the development of the SPT for a mixture of hard convex bodies in two dimensions (see Ref. 34]) is the averaged (over all possible orientations of a fluid particle of species $\nu$ ) excluded area between an inserted scaled particle $s$ with orientation $\phi_{1}$ (measured from the nematic director) and a fluid particle of species $\nu$ with orientation $\phi_{2}$, i.e.,

$$
\left\langle V_{\mathrm{excl}, \nu}^{\alpha}\right\rangle\left(L_{s}, \sigma_{s}, \phi_{1}\right)=\int d \phi_{2} h_{\nu}\left(\phi_{2}\right) V_{\mathrm{excl}, \nu}^{\alpha}\left(L_{s}, \sigma_{s}, \phi_{12}\right),
$$

where $L_{s}$ and $\sigma_{s}$ are the length and width of the scaled particle, $h_{\nu}\left(\phi_{2}\right)$ is the orientational distribution function of species $\nu$ and $\phi_{12}=\phi_{1}-\phi_{2}$ the relative angle between the axes of particles $s$ and $\nu$. The superindex $\alpha$ labels the nature of the particle, either hard rectangles $(\alpha=\mathrm{HR})$ or hard discorectangles ( $\alpha=$ HDR). The excluded area between two rectangles $(s$ and $\nu)$ is

$$
\begin{aligned}
V_{\mathrm{excl}, \nu}^{\mathrm{HR}}\left(L_{s}, \sigma_{s}, \phi_{12}\right) & =\left(L_{\nu} L_{s}+\sigma_{\nu} \sigma_{s}\right)\left|\sin \phi_{12}\right|+v_{\nu}+v_{s} \\
& +\left(L_{\nu} \sigma_{s}+L_{s} \sigma_{\nu}\right)\left|\cos \phi_{12}\right| .
\end{aligned}
$$

For hard discorectangles

$$
\begin{aligned}
V_{\mathrm{excl}, \nu}^{\mathrm{HDR}}\left(L_{s}, \sigma_{s}, \phi_{12}\right) & =L_{\nu} L_{s}\left|\sin \phi_{12}\right|+v_{\nu}+v_{s} \\
& +L_{\nu} \sigma_{s}+L_{s} \sigma_{\nu}+\frac{\pi}{2} \sigma_{\nu} \sigma_{s},
\end{aligned}
$$

where for $\mathrm{HR} v_{\beta}=L_{\beta} \sigma_{\beta}$ is the area of species $\beta$ $(\beta=\{\nu, s\})$ while for $\operatorname{HDR} v_{\beta}=L_{\beta} \sigma_{\beta}+\pi \sigma_{\beta}^{2} / 4$. The reversible work required to insert the scaled particle with fixed orientation coincides with the excess chemical potential and, in the limit of small sizes $\left(L_{s} \ll L_{\nu}, \sigma_{s} \ll \sigma_{\nu}\right.$ for any $\nu$ ), it has the following asymptotic form [19]:

$$
\begin{aligned}
\beta \mu_{\mathrm{exc}}\left(\phi_{1}\right) & \sim \mu^{(0)}\left(L_{s}, \sigma_{s}, \phi_{1}\right) \\
& \equiv-\ln \left[1-\sum_{\nu} \rho_{\nu}\left\langle V_{\mathrm{excl}, \nu}^{\alpha}\right\rangle\left(L_{s}, \sigma_{s}, \phi_{1}\right)\right]
\end{aligned}
$$

where $\rho_{\nu}$ is the density of species $\nu$ and the sum runs over all species. In the opposite limit of large sizes $\left(L_{s} \gg L_{\nu}, \sigma_{s} \gg \sigma_{\nu}\right)$ this work coincides with the thermodynamic work required to open a cavity of area $v_{s}$, which is equal to $P v_{s}$, where $P$ is the fluid pressure. The SPT interpolates between both limits using a Taylor expansion of the function $\mu^{(0)}\left(L_{s}, \sigma_{s}, \phi_{1}\right)$ around the value $\left(L_{s}, \sigma_{s}\right)=(0,0)$. The second term of this expansion is 
fixed to $P v_{s}$. Finally, all the particle lengths are taken to be those of any one of the species, say $\nu$, which results in

$$
\begin{aligned}
\beta \mu_{\mathrm{exc}, \nu}\left(\phi_{1}\right)=-\ln (1-\eta) \\
+\frac{\sum_{\tau} \rho_{\tau} \int d \phi_{2} h_{\tau}\left(\phi_{2}\right) V_{\nu \tau}^{(0)}\left(\phi_{12}\right)}{1-\eta}+\beta P v_{\nu}
\end{aligned}
$$

where $\eta=\sum \rho_{\nu} v_{\nu}$ is the total packing fraction and $V_{\nu \tau}^{(0)}\left(\phi_{12}\right)=V_{\text {excl, } \nu}^{\alpha}\left(L_{\tau}, \sigma_{\tau}, \phi_{12}\right)-v_{\nu}-v_{\tau}$. The excess chemical potential of species $\nu$ is the angular average

$$
\begin{aligned}
\beta \mu_{\mathrm{exc}, \nu} & =\int d \phi_{1} h_{\nu}\left(\phi_{1}\right)\left[\beta \mu_{\mathrm{exc}, \nu}\left(\phi_{1}\right)\right]=-\ln (1-\eta) \\
& +\frac{\sum_{\tau} \rho_{\tau}\left\langle\left\langle V_{\nu \tau}^{(0)}\right\rangle\right\rangle}{1-\eta}+\beta P v_{\nu},
\end{aligned}
$$

where $\langle\langle\cdots\rangle\rangle$ means the following double angular average:

$$
\left\langle\left\langle V_{\nu \tau}^{(0)}\right\rangle\right\rangle=\int d \phi_{1} h_{\tau}\left(\phi_{1}\right) \int d \phi_{2} h_{\nu}\left(\phi_{2}\right) V_{\nu \tau}^{(0)}\left(\phi_{12}\right) .
$$

Integrating the thermodynamic relations

$$
\frac{\partial \beta P}{\partial \rho_{\nu}}=1+\sum_{\tau} \rho_{\tau} \frac{\partial \beta \mu_{\mathrm{exc}, \tau}}{\partial \rho_{\nu}}
$$

with the use of Eq. (6) allows us to find

$$
\beta P=\frac{\rho}{1-\eta}+\frac{1}{2} \frac{\sum_{\nu \tau} \rho_{\nu} \rho_{\tau}\left\langle\left\langle V_{\nu \tau}^{(0)}\right\rangle\right\rangle}{(1-\eta)^{2}},
$$

where $\rho=\sum_{\nu} \rho_{\nu}$ is the total density. Finally, through the definition of the pressure $\beta P=\rho+\sum_{\nu} \rho_{\nu}\left[\beta \mu_{\mathrm{exc}, \nu}\right]-$ $\Phi_{\text {exc }}$, where $\Phi_{\text {exc }}=\beta \mathcal{F}_{\text {exc }} / V$ is the excess part of the free energy density in reduced units, the result (8), and Eq. (6) we obtain

$$
\Phi_{\mathrm{exc}}=-\rho \ln (1-\eta)+\frac{1}{2} \frac{\sum_{\nu \tau} \rho_{\nu} \rho_{\tau}\left\langle\left\langle V_{\nu \tau}^{(0)}\right\rangle\right\rangle}{1-\eta} .
$$
is

The ideal part of the free energy density of the mixture

$$
\Phi_{\mathrm{id}}=\sum_{\nu} \rho_{\nu}\left(\ln \rho_{\nu}-1+\int_{0}^{\pi} d \phi h_{\nu}(\phi) \ln \left[\pi h_{\nu}(\phi)\right]\right),
$$

where all distribution functions are normalized as $\int_{0}^{\pi} d \phi h_{\nu}(\phi)=1$ (note that, in view of the head-tail symmetry of the particles, the angle $\phi$ can be restricted to the interval $[0, \pi])$. The functional minimization of $\Phi=\Phi_{\text {id }}+\Phi_{\text {exc }}$ with respect to the $\left\{h_{\nu}\right\}$ allows, as usual, to find the equilibrium distribution functions and correspondingly the equilibrium free energy of the mixture.

The above theoretical scheme will be used in the following section to develop a bifurcation analysis of I-N demixing for HDR and HR. Also it will be used to calculate the phase diagram of mixtures. In order to do that we need to fix the fluid pressure, which means that the composition of one of the species and the total fluid density are no longer independent variables. Once the independent variable is chosen, the dependent variable can be calculated through the constant pressure criterion. Thus, the adequate thermodynamic potential to work with is the Gibbs free energy per particle in reduced units $\beta g=(\Phi+\beta P) / \rho$. The minimization of $\Phi$ with respect to the order parameters (in the case of the nematic phase), the condition of constant pressure and the double tangent construction on $\beta g$ with respect to the composition of one of the species, allows us to calculate the coexistence condition between different phases. Changing the pressure and repeating the above steps we have found the phase diagrams of different binary mixtures.

\section{BIFURCATION ANALYSIS}

The usual bifurcation analysis for a one-component fluid with symmetry breaking includes (i) an order parameter expansion of the free energy around the bifurcation point; (ii) the calculation of the inverse isothermal compressibility of the ordered phase at the same point. The order of the phase transition can be elucidated by the combined use of both criteria, the sign of the free-energy difference between the ordered and disordered phases, already minimized with respect to the order parameter, and the sign of the isothermal compressibility of the ordered phase, evaluated at bifurcation. If the system exhibits a tricritical point, its location can be obtained from the vanishing of either the first coefficient in the expansion of the free-energy difference or the isothermal compressibility, depending on which of them occurs first 35.

For binary mixtures fluid-fluid demixing without symmetry breaking is usually calculated from the vanishing of the determinant of the matrix with elements $\partial^{2} \Phi / \partial \rho_{i} \partial \rho_{j}$ which means that the stability of the mixture with respect to volume and composition fluctuations is violated. This allows us to obtain the demixing spinodal. However, in a symmetry-breaking transition the above matrix should be calculated from the minimized free-energy of the ordered phase using the order-parameter expansion up to the order required. This criterion is equivalent to the loss of convexity of the Gibbs free energy per particle of the ordered phase with respect to the mixture composition at the bifurcation point. Since our aim is the study of I-N demixing, which involves an orientational symmetry breaking, we will implement the latter scheme.

A Fourier-series decomposition of the orientational distribution functions

$$
h_{\nu}(\phi)=\frac{1}{\pi}\left(1+\sum_{k \geq 1} h_{k}^{(\nu)} \cos (2 k \phi)\right)
$$


should retain only even harmonics, due to the symmetry of the particles studied here $\left[h_{\nu}(\phi)=h_{\nu}(\pi-\phi)\right]$. In the neighborhood of the I-N bifurcation point we can assume that the Fourier amplitudes $\left\{h_{k}^{(\nu)}\right\}$ are small. A Taylor expansion of the difference in free energy per particle $\Delta \varphi=\varphi_{\mathrm{N}}-\varphi_{\mathrm{I}}(\varphi=\Phi / \rho)$ between $\mathrm{N}$ and I phases, up to fourth order, is therefore valid (the order is defined by a small bifurcation parameter $\left.\epsilon, h_{k}^{(\nu)} \sim \epsilon^{k}\right)$. Up to fourth order, the ideal contribution to $\Delta \varphi$ reads

$$
\begin{aligned}
\Delta \varphi_{\mathrm{id}} & \approx \sum_{\mu} x_{\mu}\left(\frac{1}{4}\left[\left(h_{1}^{(\mu)}\right)^{2}+\left(h_{2}^{(\mu)}\right)^{2}\right]\right. \\
& \left.-\frac{1}{8}\left(h_{1}^{(\mu)}\right)^{2} h_{2}^{(\mu)}+\frac{1}{32}\left(h_{1}^{(\mu)}\right)^{4}\right),
\end{aligned}
$$

whereas the excess contribution is

$$
\Delta \varphi_{\mathrm{ex}}=\frac{1}{2} y \sum_{\mu \nu} x_{\mu} x_{\nu} \frac{\Delta\left\langle\left\langle V_{\mu \nu}^{(0)}\right\rangle\right\rangle}{\langle v\rangle} .
$$

We have defined

$$
\Delta\left\langle\left\langle V_{\mu \nu}^{(0)}\right\rangle\right\rangle \equiv\left\langle\left\langle V_{\mu \nu}^{(0)}\right\rangle\right\rangle_{\mathrm{N}}-\left\langle\left\langle V_{\mu \nu}^{(0)}\right\rangle\right\rangle_{\mathrm{I}},
$$

with

$$
\frac{\Delta\left\langle\left\langle V_{\mu \nu}^{(0)}\right\rangle\right\rangle}{\langle v\rangle}=-\frac{1}{\pi} \sum_{k \geq 1} \frac{T_{\mu \nu}^{(k)}}{4 k^{2}-1} h_{k}^{(\mu)} h_{k}^{(\nu)},
$$

where it is understood that the sum is to be truncated at fourth order in the expansion parameter. In the above expresions we have also defined

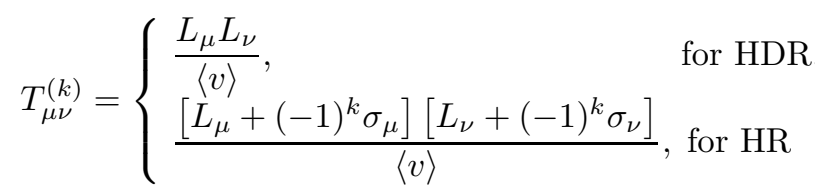

with $x_{\mu}=\rho_{\mu} / \rho$ being the molar fraction of species $\mu$, $y=\eta /(1-\eta)$, and $\langle v\rangle=\sum_{\nu} x_{\nu} v_{\nu}$ the average particle area of the mixture. The complete free-energy expansion up to fourth order reads

$$
\begin{aligned}
\Delta \varphi & =a_{11}^{(1)}\left(h_{1}^{(1)}\right)^{2}+a_{22}^{(1)}\left(h_{1}^{(2)}\right)^{2}+2 a_{12}^{(1)} h_{1}^{(1)} h_{1}^{(2)} \\
& +a_{11}^{(2)}\left(h_{2}^{(1)}\right)^{2}+a_{22}^{(2)}\left(h_{2}^{(2)}\right)^{2}+2 a_{12}^{(2)} h_{2}^{(1)} h_{2}^{(2)} \\
& +a_{11}^{(3)}\left(h_{1}^{(1)}\right)^{2} h_{2}^{(1)}+a_{22}^{(3)}\left(h_{1}^{(2)}\right)^{2} h_{2}^{(2)}+a_{11}^{(4)}\left(h_{1}^{(1)}\right)^{4} \\
& +a_{22}^{(4)}\left(h_{1}^{(2)}\right)^{4} .
\end{aligned}
$$

The expressions for the $a_{\mu \nu}^{(k)}$ 's are

$$
\begin{aligned}
& a_{\mu \nu}^{(k)}=\frac{x_{\mu}}{4}\left(\delta_{\mu \nu}-\frac{2 y x_{\nu}}{\left(4 k^{2}-1\right) \pi} T_{\mu \nu}^{(k)}\right), k=1,2, \\
& a_{\mu \mu}^{(3)}=-\frac{x_{\mu}}{8}, \quad a_{\mu \mu}^{(4)}=\frac{x_{\mu}}{32}, \quad \mu, \nu=1,2,
\end{aligned}
$$

where $\delta_{\mu \nu}$ is the Kronecker delta.

Minimizing $\Delta \varphi$ with respect to all the amplitudes except one (say $h_{1}^{(1)}$ ), substituting the results for the other amplitudes in (17), and neglecting all terms with order higher than four, we obtain an effective free energy difference as

$$
\Delta \varphi=A\left(h_{1}^{(1)}\right)^{2}+B\left(h_{1}^{(1)}\right)^{4},
$$

with the following explicit expressions for the coefficients $A$ and $B$ :

$$
\begin{aligned}
A & =a_{11}^{(1)}-\frac{\left(a_{12}^{(1)}\right)^{2}}{a_{22}^{(1)}} \\
B & =a_{11}^{(4)}+\left(a_{22}^{(4)}-\frac{\left(a_{22}^{(3)}\right)^{2}}{4 a_{22}^{(2)}}\right)\left(\frac{a_{12}^{(1)}}{a_{22}^{(1)}}\right)^{4} \\
& -\frac{\left[a_{11}^{(3)}-a_{22}^{(3)} \frac{a_{12}^{(2)}}{a_{22}^{(2)}}\left(\frac{a_{12}^{(1)}}{a_{22}^{(1)}}\right)^{2}\right]^{2}}{4\left(a_{11}^{(2)}-\frac{\left(a_{12}^{(2)}\right)^{2}}{a_{22}^{(2)}}\right)}
\end{aligned}
$$

Note that $A$ and $B$ depend on $x=x_{2}$ and $y$. To proceed we must bear in mind that demixing phase transitions usually imply fractionation (different composition $x$ of the coexisting phases) as well as a change in packing fraction $\eta$. Therefore, we should expand both the composition $x$ and the variable $y$ around the bifurcation point $\left(x^{*}, y^{*}\right)$

$$
x \approx x^{*}+x^{(2)}\left(h_{1}^{(1)}\right)^{2}, \quad y \approx y^{*}+y^{(2)}\left(h_{1}^{(1)}\right)^{2} .
$$

Inserting the expansions (231) in the first derivative of (20) with respect to $h_{1}^{(1)}$ and equating the result to zero, order by order, we obtain the following conditions:

$$
\begin{gathered}
A^{*}=0, \quad \mathcal{O}\left[h_{1}^{(1)}\right], \\
A_{x}^{*} x^{(2)}+A_{y}^{*} y^{(2)}=-2 B^{*}, \quad \mathcal{O}\left[\left(h_{1}^{(1)}\right)^{3}\right],
\end{gathered}
$$

where $f^{*} \equiv f\left(x^{*}, y^{*}\right)$ with $\left(f=A, B, A_{x}, A_{y}\right)$, and the subindices $x, y$ in Eq. (25) mean the partial derivatives of $A$ with respect to $x$ and $y$, respectively. Solving Eq. (24) gives the packing fraction as a function of composition at the bifurcation point, whereas Eq. 251) allows to find a relation between $x^{(2)}$ and $y^{(2)}$, respectively.

Expanding (20) up to fourth order around the bifurcation point and using (24) and (25) we obtain the energy difference as

$$
\Delta \varphi=-B^{*}\left(h_{1}^{(1)}\right)^{4} .
$$


Depending on the sign of $B^{*}$ the nematic branch bifurcates below (positive sign) or above (negative sign) the isotropic branch. The latter case corresponds to a firstorder transition. Using (23) and (25), Eq. (26) can be rewritten as

$$
\varphi_{\mathrm{N}}=\varphi_{\mathrm{I}}-\frac{1}{4 B^{*}}\left[A_{x}^{*}\left(x-x^{*}\right)+A_{y}^{*}\left(y-y^{*}\right)\right]^{2} .
$$

The stability of the mixture with respect to volume and composition fluctuations is guaranteed when

$$
\left(\frac{\partial^{2} \Phi}{\partial \rho_{1}^{2}}\right)\left(\frac{\partial^{2} \Phi}{\partial \rho_{2}^{2}}\right)-\left(\frac{\partial^{2} \Phi}{\partial \rho_{1} \partial \rho_{2}}\right)^{2}>0
$$

Fixing the areas of all species to one (in this case the demixing criterion will depend on the difference in particle shapes and not on their areas) the Eq. (28), in terms of the variables $(x, y)$, can be written as

$$
H \equiv \frac{(1+y)^{4}}{y^{2}}\left[\frac{\partial}{\partial y}\left(y^{2} \frac{\partial \varphi}{\partial y}\right) \frac{\partial^{2} \varphi}{\partial x^{2}}-\left(y \frac{\partial^{2} \varphi}{\partial x \partial y}\right)^{2}\right]>0
$$

which, for the $\mathrm{N}$ phase and at the bifurcation point [with use of (27)], becomes

$$
\begin{gathered}
H_{\mathrm{N}}^{*}=H_{\mathrm{I}}^{*}-\frac{\left(1+y^{*}\right)^{4}}{2\left(y^{*}\right)^{2} B^{*}}\left\{\left[\frac{\partial}{\partial y}\left(y^{2} \frac{\partial \varphi_{\mathrm{I}}}{\partial y}\right)\right]^{*}\left(A_{x}^{*}\right)^{2}\right. \\
\left.-2\left(\frac{\partial^{2} \varphi_{\mathrm{I}}}{\partial x \partial y}\right)^{*}\left(y^{*}\right)^{2} A_{x}^{*} A_{y}^{*}+\left(\frac{\partial^{2} \varphi_{\mathrm{I}}}{\partial x^{2}}\right)^{*}\left(y^{*} A_{y}^{*}\right)^{2}\right\}>0 .
\end{gathered}
$$

It is easy to show that $H_{\mathrm{I}}(x, \eta)>0$ for HDR, freely rotating $\mathrm{HR}$ and $\mathrm{HR}$ in the Zwanzig approximation. This result confirms the general wisdom [25] mentioned above on the stability of a two-dimensional mixture of hard bodies against I-I fluid demixing, in the framework of the SPT. However, fixing the particle orientations to be parallel we obtain the condition for Eq. (28) to be violated as

$$
\eta \geq \eta^{*} \equiv\left[1+x(1-x)\left(\tau-\tau^{-1}\right)^{2}\right]^{-1 / 2}
$$

with

$$
\tau= \begin{cases}\sqrt{\frac{\kappa_{2}-1+\frac{\pi}{4}}{\kappa_{1}-1+\frac{\pi}{4}}}, & \text { for HDR } \\ \sqrt{\frac{\kappa_{2}}{\kappa_{1}}}, & \text { for HR }\end{cases}
$$

and, correspondingly, the position of the critical point in the pressure-composition plane is

$$
x_{c}=\frac{1}{2}, \quad \beta P_{c} v_{1}=\frac{3\left(\tau+\tau^{-1}\right)-2}{\left(\tau+\tau^{-1}-2\right)^{2}},
$$

where $\kappa_{\nu}=\left(L_{\nu}+\sigma_{\nu}\right) / \sigma_{\nu}$ for $\operatorname{HDR}$ while $\kappa_{\nu}=L_{\nu} / \sigma_{\nu}$ for HR (remember that these results apply to mixtures such that all particle areas have been taken to be unity). This result confirms that there exists N-N demixing in the approximation that particles are perfectly aligned.

The condition $H_{\mathrm{N}}^{*}=0$ will be used in the next section to calculate the I-N demixing region in the plane $\lambda-x_{1}^{*}$, where $\lambda=\kappa_{2} / \kappa_{1}$ and $\kappa_{\nu}$ is the aspect ratio of species $\nu$, and it is equivalent to the condition that the Gibbs free energy per particle of the $\mathrm{N}$ phase have zero curvature (second derivative with respect to $x_{1}$ ) at bifurcation, thereby determining the I-N demixing tricritical point.

\section{RESULTS FROM THE BIFURCATION ANALYSIS}

This section shows the results from the bifurcation analysis, whose formalism was introduced in the preceding section. We have divided this section into three sections. The first two are devoted to the study of the freely-rotating HDR and HR models, respectively, while in the third section we discuss the implementation of the formalism to the restricted-orientation model (Zwanzig model). In this last section results from the calculation of the exact phase diagrams of this model are shown. The main purpose is to check the results from the bifurcation analysis against exact calculations (note that there is no need to use any parametrization in the Zwanzig model).

\section{A. Hard discorectangles}

The solution of Eq. 24 for HDR allows us to write the packing fraction value at the bifurcation point as a function of the composition of the mixture,

$$
\eta^{*}=\left(1+\frac{2}{3 \pi} \frac{\left\langle L^{2}\right\rangle}{\langle v\rangle}\right)^{-1}
$$

where we have defined $\left\langle u^{n}\right\rangle=\sum_{\nu} x_{\nu}^{*} u_{\nu}^{n}$ for a generic quantity $u$. The expression for $B^{*}$ which, as pointed out before, defines the relative positions of the $\mathrm{I}$ and $\mathrm{N}$ branches near the bifurcation point, is

$$
B^{*}=\frac{x_{1}^{*}}{4^{4} z_{1}}\left[z_{2}\left(3+z_{1}\right) r^{2}-2 z_{1} z_{2} r+z_{1}\left(3+z_{2}\right)\right],
$$

where $r=L_{2} / L_{1}$ and $z_{\nu}=x_{\nu}^{*} L_{\nu}^{2} /\left\langle L^{2}\right\rangle<1$. The quadratic polynomial $P(r)$ enclosed by the square brackets has a discriminant $D=-48 z_{1} z_{2}$, which obviously is always negative, while $P(0)>0$. Thus the coefficient $B^{*}$ is always positive and, as a consequence, the nematic branch always bifurcates from below with respect to the isotropic one.

We take the areas of all particles to be equal to 1 , which means that the particle length and width, in units of $v_{\mu}^{1 / 2}$, are

$$
\sigma_{\mu}=\left(\kappa_{\mu}-1+\frac{\pi}{4}\right)^{-1 / 2}, \quad L_{\mu}=\left(\kappa_{\mu}-1\right) \sigma_{\mu} .
$$




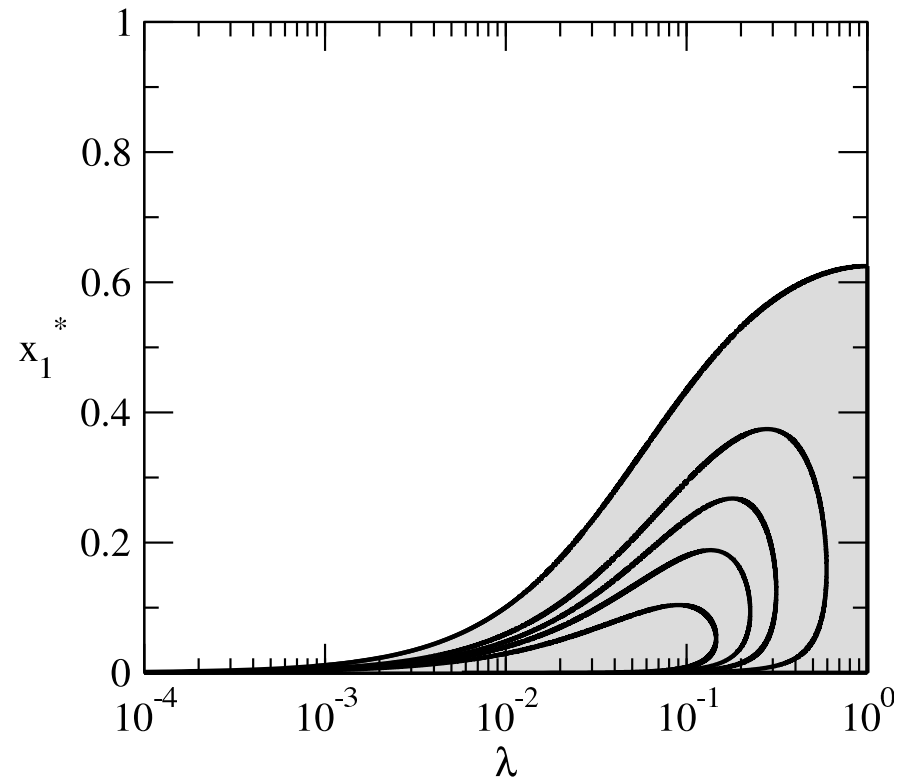

FIG. 1: Solutions of $H_{\mathrm{N}}^{*}=0$ in the $x_{1}^{*}$ (composition)- $\lambda$ plane, where $\lambda=\kappa_{2} / \kappa_{1}$, in logarithmic scale, for HDR mixtures with particle areas equal to unity. The curves are shown for different values of $\kappa_{2}$. The shaded areas represent the demixing regions predicted from $H_{\mathrm{N}}^{*}<0$. From outside to inside the values of $\kappa_{2}$ are $1,2,3,5$, and $\infty$ (the Onsager limit).

Inserting (34) and (35) in (30), the final expression for $H_{\mathrm{N}}^{*}$ can be written in a particularly compact form,

$$
\begin{aligned}
H_{\mathrm{N}}^{*} & =\frac{\left(1+y^{*}\right)^{4}}{x_{1}^{*} x_{2}^{*}\left(y^{*}\right)^{2}}\left[4+\frac{3 \pi}{\left\langle L^{2}\right\rangle}\right. \\
& \left.-\frac{8}{3} s_{3}^{2}\left(1+s_{2}^{2}+\frac{3}{4} \frac{\langle p\rangle^{2}}{\left\langle L^{2}\right\rangle}\left(s_{2}-s_{1}\right)^{2}\right)\right], \\
s_{1} & =\sqrt{\frac{\left\langle p^{2}\right\rangle}{\langle p\rangle^{2}}-1}, \quad s_{2}=\sqrt{\frac{\left\langle L^{4}\right\rangle}{\left\langle L^{2}\right\rangle^{2}}-1} \\
s_{3} & =\sqrt{\frac{3\left\langle L^{2}\right\rangle^{3}}{4\left\langle L^{4}\right\rangle\left\langle L^{2}\right\rangle-\left\langle L^{3}\right\rangle^{2}}},
\end{aligned}
$$

where $p=2 L+\pi \sigma$ is the perimeter of the particle.

The equality to zero of the expression enclosed by square brackets in (37), for the specific case $L_{2}=0$ (or $\kappa_{2}=1$, i.e. a binary mixture of hard disks and HDR), gives us the analytic solution $x_{1}^{*}=15 \pi / 4\left(6 \pi+L_{1}^{2}\right)$, shown in Fig. 1 as the curve which encloses all the other curves in the $\lambda-x_{1}^{*}$ plane $\left(\lambda=\kappa_{2} / \kappa_{1}\right)$. Note that the above expression is not valid when $\lambda=1$ or $x_{1}^{*}=0$ (the onecomponent hard disk fluid). In Fig. [1 the solutions of $H_{\mathrm{N}}^{*}=0$ for other mixtures, including the Onsager limit (i.e. a mixture of hard needles), are shown. It is remarkable that, even in this limit, the mixture can demix. To calculate these curves the aspect ratio $\kappa_{2}$ was fixed, while $\kappa_{1}$ was varied from 1 to $10^{4}$, and $H_{\mathrm{N}}^{*}=0$ was solved for $x_{1}^{*}$. The curve corresponding to the Onsager limit $\kappa_{\mu} \rightarrow \infty(\mu=1,2)$ was calculated by first tak-

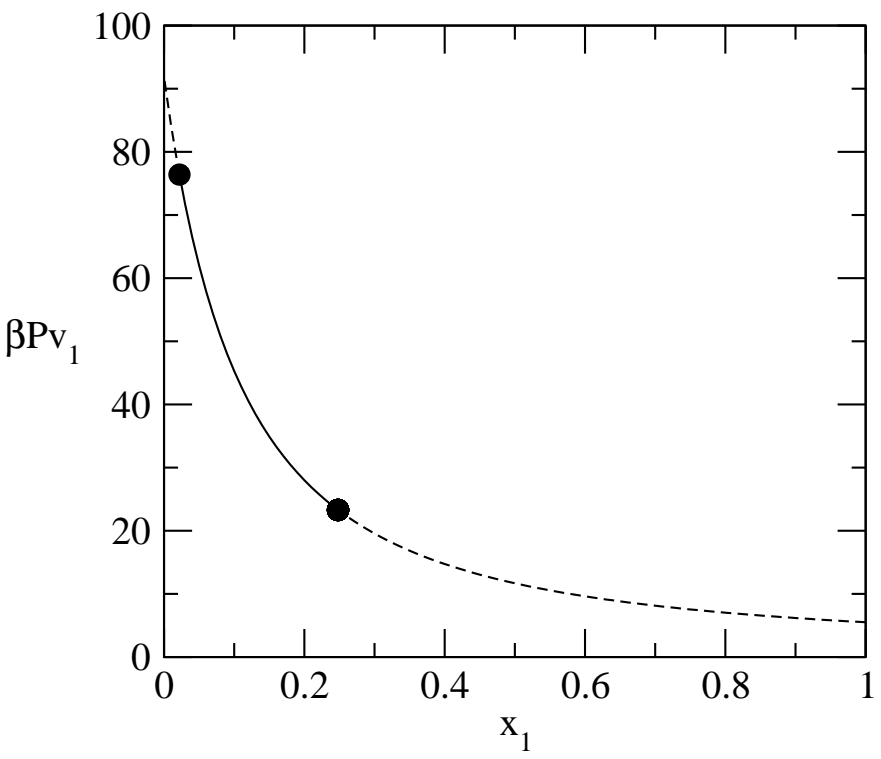

FIG. 2: Reduced pressure $\beta P v_{1}$ versus composition $x_{1}$ for a binary mixture of HDR with $\kappa_{1}=5$ and $\kappa_{2}=2$. Filled circles indicate the intersections of a vertical line located at the corresponding value of $\lambda=2 / 5$ and the solution of $H_{\mathrm{N}}^{*}=0$ in Fig. 1] The continuous line is the portion corresponding to the shaded region in the latter figure.

ing the corresponding limit of the expression enclosed by square brackets in Eq. (37) (which depends only on $\lambda$ ) and solving again for $x_{1}^{*}$.

We now proceed to explain the physics behind the behavior shown in Fig. 1 using the particular values $\lambda=2 / 5, \kappa_{2}=2$ as an example. A vertical line located at this value of $\lambda$ intersects the curve corresponding to $\kappa_{2}=2$ at two points, giving two values of $x_{1}^{*}$ at which $H_{\mathrm{N}}^{*}=0$. In Fig. 2 we plot the reduced pressure as a function of $x_{1}$ for these values of $\lambda$ and $\kappa_{2}$. As can be seen from this figure the pressure is a monotonically decreasing function of $x_{1}$, which means that the abovementioned intersection with a higher value of $x_{1}^{*}$ has a lower pressure than the other one. This, in turn, implies that the first point corresponds to a genuine tricritical point where, for the first time, the Gibbs free energy loses its convexity with respect to the composition variable. The second point also corresponds to a loss of convexity of the Gibbs free energy but, as will be discussed later, lies inside the two-phase region of a demixing transition.

\section{B. Hard rectangles}

As shown in Refs. 33] and [34 the system of HR exhibits a transition to a phase with fourfold symmetry, the so-called tetratic phase $\left(\mathrm{N}_{t}\right)$. In this phase the orientational distribution function has a symmetry under rotation by $\pi / 2, h(\phi)=h(\pi / 2-\phi)$. The $\mathrm{I}^{-\mathrm{N}_{t}}$ transition was always found to be of second order in the whole region of its stability $(1 \leq \kappa \leq 2.21)$. Thus the ques- 
tion naturally arises as to the relative stability of this phase in the binary mixture. The above symmetry dictates that the odd Fourier amplitudes $\left\{h_{2 j-1}^{(\nu)}\right\}$ should be equal to zero. Using this constraint and carrying out the same expansion for the free-energy difference around the bifurcation point, we arrive at the same Eq. (17), with the coefficients $a_{\mu \nu}^{(k)}(k=1,2)$ obtained from Eq. (18), but through the substitution $k \rightarrow 2 k$ on the right-hand side of (18), while for $k=3,4$ the coefficients are given by the same expression (19). The packing fraction at the I- $\mathrm{N}_{t}$ bifurcation point, obtained as the solution of (24), is

$$
\eta_{\mathrm{N}_{\mathrm{t}}}^{*}=\left(1+\frac{2}{15 \pi} \frac{\left\langle(L+\sigma)^{2}\right\rangle}{\langle v\rangle}\right)^{-1}
$$

while the value corresponding to the isotropic-uniaxial nematic $\left(\mathrm{N}_{u}\right)$ bifurcation point, calculated directly from (18), (19), and (24), is

$$
\eta_{\mathrm{N}_{\mathrm{u}}}^{*}=\left(1+\frac{2}{3 \pi} \frac{\left\langle(L-\sigma)^{2}\right\rangle}{\langle v\rangle}\right)^{-1} .
$$

The equality of (40) and (41) defines a line in the plane $x_{1}^{*}-\kappa_{1}$ (fixing $\kappa_{2}$ ) where the I- $\mathrm{N}_{t}$ transition preempts the I-N $\mathrm{N}_{u}$ transition. Taking the areas of both particles to be unity, as was done for HDR, and changing to variables $\theta_{\nu}=\ln \left(\kappa_{\nu}\right) / 2(\nu=1,2)$, we obtain the solution of $\eta_{\mathrm{N}_{\mathrm{t}}}=$ $\eta_{\mathrm{N}_{\mathrm{u}}}$ as

$$
x_{1}^{*}=\frac{1-4 \sinh ^{2} \theta_{2}}{4\left(\sinh ^{2} \theta_{1}-\sinh ^{2} \theta_{2}\right)},
$$

with the constraint $\sinh ^{2} \theta_{\mu} \leq 1 / 4 \leq \sinh ^{2} \theta_{\nu}$ (and $\mu \neq$ $\nu)$.

This result should be taken with some care because the $\mathrm{I}_{-} \mathrm{N}_{\alpha}(\alpha=u, t)$ transitions can be of first order and the relative position of binodals can change the scenario predicted above. In order to elucidate the nature of these transitions, we have first calculated the coefficient $B_{\mathrm{N}_{\mathrm{t}}}^{*}$ for the tetratic phase which results in

$B_{\mathrm{N}_{\mathrm{t}}}^{*}=\frac{x_{1}^{*}}{4^{5} z_{1}}\left[z_{2}\left(11+5 z_{1}\right) r^{2}-10 z_{1} z_{2} r+z_{1}\left(11+5 z_{2}\right)\right]$,

where $r=\cosh \theta_{2} / \cosh \theta_{1}$ and $z_{\mu}=$ $x_{\mu}^{*} \cosh ^{2} \theta_{\mu} /\left\langle\cosh ^{2} \theta\right\rangle$. Again the second-order polynomial with respect to $r$ enclosed by the square brackets is always greater than zero. Thus $B^{*}>0$ and the $\mathrm{N}_{t}$ energy branch bifurcates from below from the I branch.
In a second step we have calculated $H_{\mathrm{N}_{\mathrm{t}}}^{*}$ which gives

$$
\begin{aligned}
H_{\mathrm{N}_{\mathrm{t}}}^{*} & =16 \frac{\left(1+y^{*}\right)^{4}}{x_{1}^{*} x_{2}^{*}\left(y^{*}\right)^{2}}\left[1-\frac{2}{11} s_{3}^{2}\right. \\
& \left.\times\left(1+s_{2}^{2}+15 \frac{\left(s_{2}-s_{1}\right)^{2}}{1+s_{1}^{2}}\right)\right], \\
s_{k} & =\sqrt{\frac{\left\langle\cosh ^{2 k} \theta\right\rangle}{\left\langle\cosh ^{k} \theta\right\rangle^{2}}-1, \quad k=1,2,} \\
s_{3} & =\sqrt{\frac{11\left\langle\cosh ^{2} \theta\right\rangle^{3}}{16\left\langle\cosh ^{4} \theta\right\rangle\left\langle\cosh ^{2} \theta\right\rangle-5\left\langle\cosh ^{3} \theta\right\rangle^{2}}} .
\end{aligned}
$$

The region in the $x_{1}^{*}-\kappa_{1}$ plane where $H_{\mathrm{N}_{\mathrm{t}}}^{*}<0$ defines the demixing region, bounded by a dashed line in the inset of Fig. 31(a) for $\kappa_{2}=1$. As can be seen this region is closed as in the case of HDR for the I-N transition. The main reason for this behavior is that in the onecomponent limit the $\mathrm{I}-\mathrm{N}_{t}$ transition is of second order $\left(B_{\mathrm{N}_{\mathrm{t}}}^{*}>0\right.$ and $\left[\left(\varkappa_{T}\right)_{\mathrm{N}_{\mathrm{t}}}^{-1}\right]^{*}>0[\underline{36}]$ for all $\left.\kappa_{1}\right)$ which in turn means that the solutions of $H_{\mathrm{N}_{\mathrm{t}}}\left(x_{1}^{*}, \kappa_{1}\right)=0$ do not intersect the lines $x_{1}^{*}=\{0,1\}$.

As the third and last step we have calculated the expressions for $B_{\mathrm{N}_{\mathrm{u}}}^{*}$ and $H_{\mathrm{N}_{\mathrm{u}}}^{*}$, which are too large to be shown here, except for their one-component limits $x_{1}^{*} \rightarrow 1$,

$$
\begin{aligned}
\lim _{x_{1}^{*} \rightarrow 1} B_{\mathrm{N}_{\mathrm{u}}}^{*} & =\frac{1}{64} \frac{5-2 \operatorname{coth}^{2} \theta_{1}}{5-\operatorname{coth}^{2} \theta_{1}}, \\
{\left[\left(\varkappa_{T}\right)_{\mathrm{N}_{\mathrm{u}}}^{-1} v_{1}\right]^{*} } & =y^{*}\left(1+y^{*}\right) \frac{15 \operatorname{coth}^{2} \theta_{1}-6 \operatorname{coth}^{4} \theta_{1}-5}{5-2 \operatorname{coth}^{2} \theta_{1}},
\end{aligned}
$$

where, rather than $H$, the inverse isothermal compressibility $\varkappa_{T}^{-1}$ was chosen as the adequate thermodynamic variable to elucidate the nature of the $\mathrm{I}-\mathrm{N}_{u}$ transition of a one-component fluid [36]. An important difference to be mentioned is that, for $\mathrm{HR}, B_{\mathrm{N}_{u}}^{*}$ in the one-component limit can be negative for some $\kappa_{1}$ [see Eq. [47)], which indicates the presence of a tricritical point. In this limit the condition $\left[\left(\varkappa_{T}\right)_{\mathrm{N}_{\mathrm{u}}}^{-1}\right]^{*}=0$ is more stringent to determine the exact location of this point which gives, equating the numerator of the right-hand side of Eq. (48) to zero, the solution

$$
\kappa_{1}^{*}=\frac{\sqrt{1+\sqrt{7 / 15}}+2 / \sqrt{5}}{\sqrt{1+\sqrt{7 / 15}}-2 / \sqrt{5}} \approx 5.44
$$

[34]. On the other hand, under the assumption that both the $\mathrm{I}-\mathrm{N}_{u}$ and $\mathrm{I}-\mathrm{N}_{t}$ are of second order, the transition to the $\mathrm{N}_{t}$ phase preempts the $\mathrm{I} \mathrm{N}_{u}$ transition for $\kappa_{1}$ less than a value $\kappa_{1}^{*}$ obtained from Eq. (42) by taking the one-component limit $x_{1}^{*}=1$. The result is $\kappa_{1}^{*}=(3+\sqrt{5}) / 2 \approx 2.62$. However, for this value of $\kappa_{1}^{*}$, 

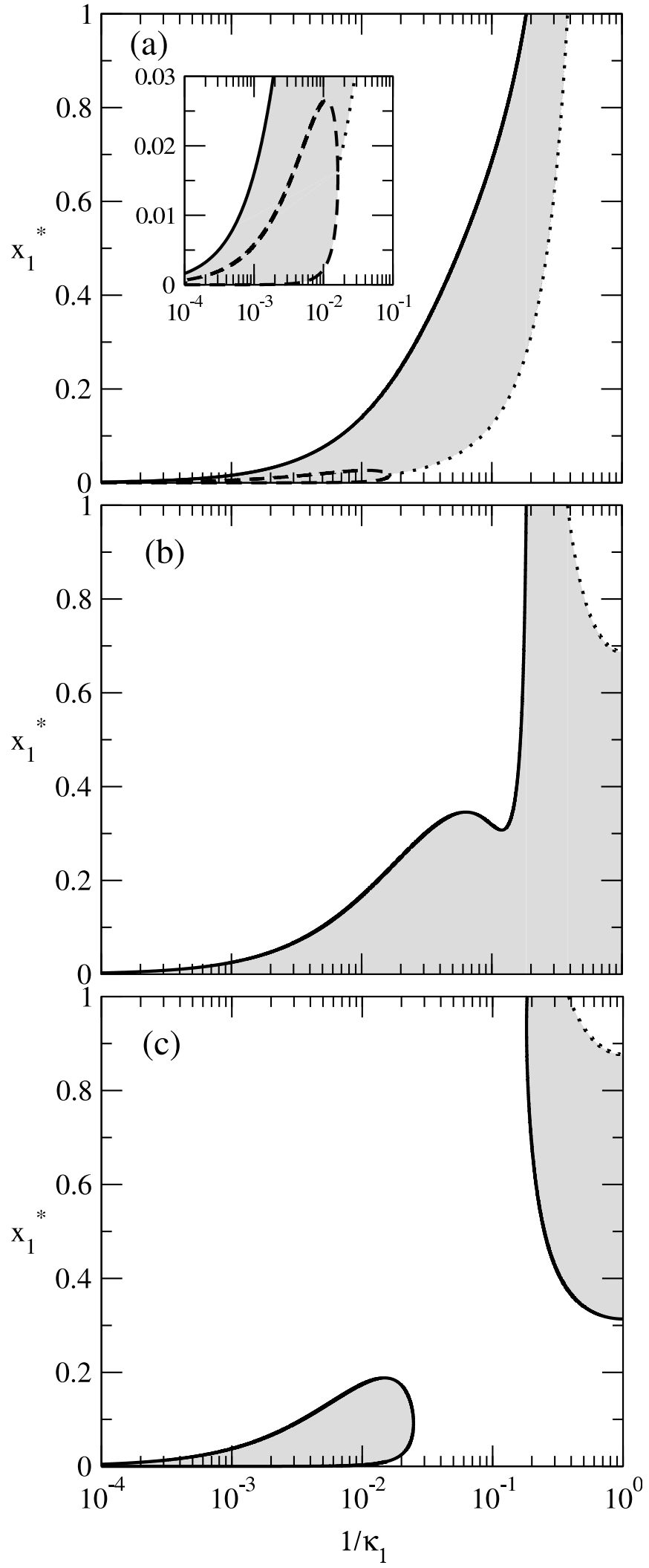

FIG. 3: Demixing regions predicted from $H_{\mathrm{N}_{\alpha}}^{*}=0(\alpha=u, t)$ for HR in the $x_{1}^{*}-\kappa_{1}$ plane. The solutions of the equations $H_{\mathrm{N}_{\mathrm{u}}}^{*}=0$ (solid line), $H_{\mathrm{N}_{\mathrm{t}}}^{*}=0$ (dashed line), and the function (42) (dotted lines) are shown for $\kappa_{2}=1$ (a), 5 (b), and 10 (c). the I- $\mathrm{N}_{u}$ transition is of first order, so that the value of $\kappa_{1}^{*}$ that determines when the $\mathrm{N}_{t}$ phase begins to be stable (for $\kappa_{1}<\kappa_{1}^{*}$ ) should be found as the intersection of the I- $\mathrm{N}_{t}$ spinodal with the I binodal of the $\mathrm{I}^{-} \mathrm{N}_{u}$ coexistence. This value turns out to be 33$] \kappa_{1}^{*}=2.21$. Thus, in the one-component limit, the range where the transition from the I phase to an orientational ordered phase is of first order is $2.21 \lesssim \kappa_{1} \lesssim 5.44$. For the mixture, the more stringent condition turns out to be $H_{\mathrm{N}_{\mathrm{u}}}^{*}\left(x_{1}^{*}, \kappa_{1}\right)=0$ whose solutions are shown for $\kappa_{2}=1,5$, and 10 (see Fig. 3). The main differences between these figures and that obtained for HDR can be summarized as follows: (i) Some of the predicted demixing regions shown in Fig. 3 are open due to the first order nature of the $\mathrm{I}_{-} \mathrm{N}_{u}$ transition in the one-component limit, as was discussed above. In contrast the solution to $H_{\mathrm{N}_{\mathrm{t}}}^{*}=0$ generates a closed loop due to the second order nature of the $\mathrm{I}_{-} \mathrm{N}_{t}$ transition [see the inset of Fig. 3(a)]. Finally for $\kappa=10$ [Fig. 3(c)] $H_{\mathrm{N}_{\mathrm{u}}}^{*}=0$ has two separate solutions, one of which is closed, the other one bounding an open region. In the Onsager limit we again obtain the closed loop shown in Fig. 11 as should be expected, since both models have the same asymptotic limit. (ii) Due to the presence of the $\mathrm{N}_{t}$ phase in the mixture of $\mathrm{HR}$, one of the curves which bounds the demixing regions is given by Eq. (42) (the dotted lines in Fig. [3). (iii) The demixing regions for HR mixture are in general wider as compared to those of HDR.

Let us discuss the behavior of the pressure, using Fig. 31 a) and, as an example, the case $\kappa_{2}=1$ and $\kappa_{1}=10^{2}$. This behavior is similar to that shown in Fig. 2 for the HDR model, namely the pressure is a monotonically decreasing function of composition. Also there are two points obtained from the intersection of the vertical line at $\kappa_{1}^{-1}=10^{-2}$ and the boundaries of the shaded region (the upper one on the solid line and the other on the lower branch of the dashed line). These in principle would correspond to tricritical points as in the case of HDR. The point corresponding to a lower pressure (higher composition) is a genuine tricritical point while the other one might be inside $\mathrm{I}_{-} \mathrm{N}_{u}$ or $\mathrm{I}-\mathrm{N}_{t}$ demixing regions.

\section{The Zwanzig model}

This simple model, as applied to HR, allows the calculation of the phase diagram without any parametrization because the orientational distribution function can be taken as $h_{\mu}(\phi)=\left[\left(1+q_{\mu}\right) / 2\right] \delta(\phi)+\left[\left(1-q_{\mu}\right) / 2\right] \delta(\phi-$ $\pi / 2)[\delta(x)$ is the Dirac delta function], corresponding to a binary mixture of two species with perpendicular orientations. Thus, the excess part of the free energy is a second degree polynomial of the order parameters $q_{\nu}$ (with $-1 \leq q_{\nu} \leq 1$ ), and the minimization of the total free energy requires to solve two transcendental equations to find their equilibrium values. Since no parametrization is necessary, the location of all tricritical points, as obtained from the minimization, are exactly the same as 
those obtained from the bifurcation analysis. This in fact is the reason why we have chosen to use this model.

Once we calculate the phase diagram we can compare the results to those obtained using the bifurcation analysis. To implement the latter we need the following expressions:

$$
\begin{aligned}
& a_{\mu \nu}^{(1)}=\frac{x_{\mu}}{2}\left(\delta_{\mu \nu}-2 y x_{\nu} \sinh \theta_{\mu} \sinh \theta_{\nu}\right), \\
& a_{\mu \nu}^{(2)}=a_{\mu \mu}^{(3)}=0, \quad a_{\mu \mu}^{(4)}=\frac{x_{\mu}}{12},
\end{aligned}
$$

which allows, using the same procedure described above, to find the packing fraction at the bifurcation point,

$$
\eta^{*}=\left(1+2\left\langle\sinh ^{2} \theta\right\rangle\right)^{-1},
$$

and the coefficient $B^{*}$,

$$
B^{*}=\frac{1}{12} \frac{\left\langle\sinh ^{4} \theta\right\rangle}{\sinh ^{4} \theta_{1}}>0,
$$

where the constraint of all particle areas being equal to 1 was imposed and the same change of variables $\left[\theta_{\mu}=\ln \left(\kappa_{\mu}\right) / 2\right]$ was used. Finally, to find the demixing behavior of this model, we need to make the expression

$$
\begin{aligned}
H_{\mathrm{N}}^{*} & =\frac{\left(1+y^{*}\right)^{4}}{x_{1}^{*} x_{2}^{*}\left(y^{*}\right)^{2}}\left[1+a-\frac{3}{2}\left(1+s_{3}^{2}\right)^{-1}\right. \\
& \left.\times\left(1+\left(a s_{2}\right)^{2}+a \frac{\left(a s_{2}-s_{1}\right)^{2}}{1+s_{1}^{2}}\right)\right], \\
s_{k} & =\sqrt{\frac{\left\langle\cosh ^{2 k} \theta\right\rangle}{\left\langle\cosh ^{k} \theta\right\rangle^{2}}-1}, \quad k=1,2 \\
s_{3} & =\sqrt{\frac{\left\langle\sinh ^{4} \theta\right\rangle}{\left\langle\sinh ^{2} \theta\right\rangle^{2}}-1,} \quad a=\frac{\left\langle\cosh ^{2} \theta\right\rangle}{\left\langle\sinh ^{2} \theta\right\rangle}
\end{aligned}
$$

to vanish. The solutions of this equation for different values of $\kappa_{2}$ are shown in Fig. 4 As can be seen the general topology is similar to that found for HDR. Comparing both figures (1) and 4) we can draw as a conclusion that the discretization of orientations unfavors the I-N demixing.

The phase diagram of a Zwanzig binary mixture of species with aspect ratios $\kappa_{\mu}$ equal to $9(\mu=1)$ and 2 $(\mu=2)$ is shown in Fig. [5 in the pressure-composition plane. The tricritical point (where the $\mathrm{I}_{-} \mathrm{N}_{1}$ demixing transition changes from first to second order as the pressure is reduced) at $x_{1}^{*}=0.1034$ and $\beta P^{*} v_{1}=5.2928$ coincides exactly with the point predicted from the bifurcation analysis (see in Fig. [4 the upper intersection of the curve for $\kappa_{2}=2$ with a vertical line located at $\lambda=2 / 9 \approx 0.222$ ).

An important feature of this phase diagram is that the binodals of the I- $\mathrm{N}_{1}$ or $\mathrm{N}_{1}-\mathrm{N}_{2}$ transitions tend to the one-component asymptotes $x_{1}=0$ and $x_{1}=1$ as the pressure is increased. This trend, characteristic of demixing scenarios (such as the usual I-I fluid demixing),

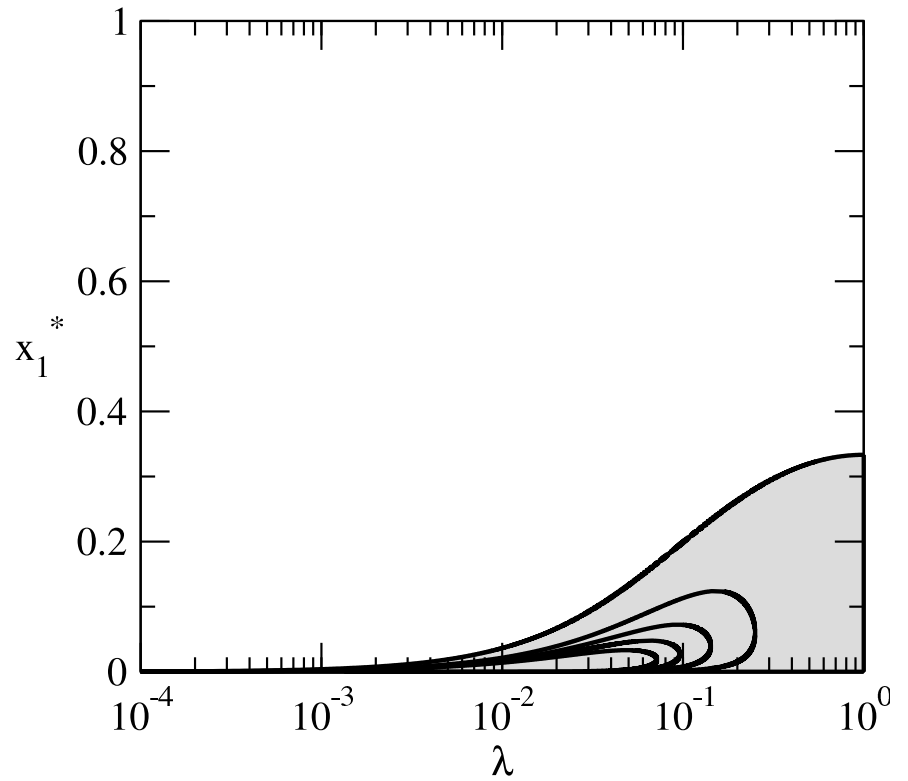

FIG. 4: Demixing regions (shaded areas) of the Zwanzig model predicted from $H_{\mathrm{N}}^{*}=0$. Different curves are the solutions of (53) for $\kappa_{2}=1,2,3,5$, and $\infty$ (the Onsager limit) from outside to inside.

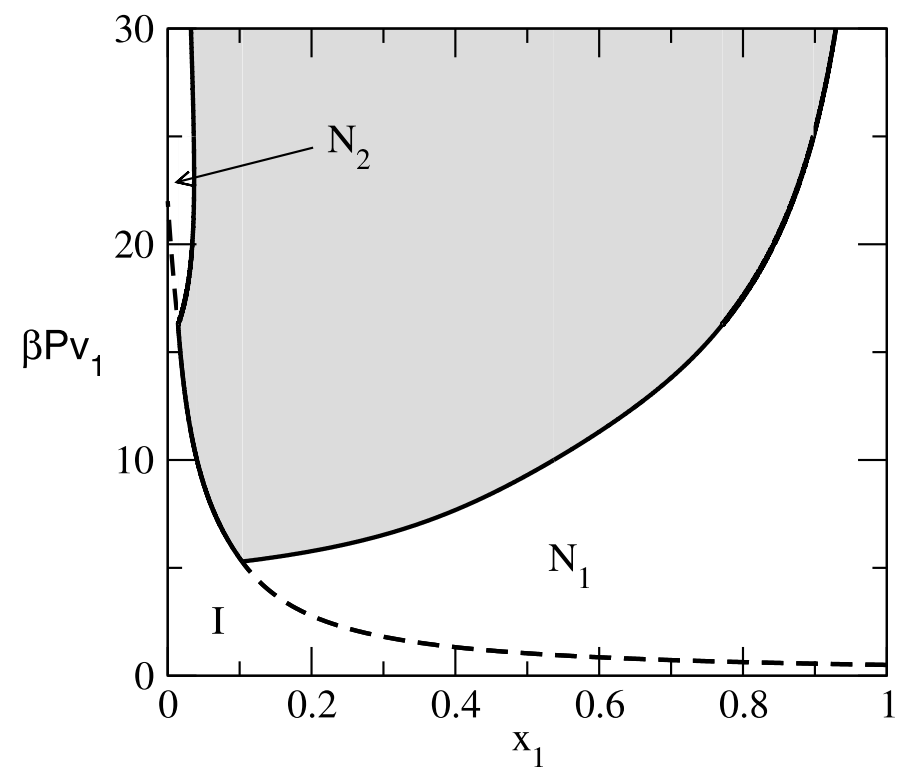

FIG. 5: Phase diagram of the Zwanzig mixture with $\kappa_{1}=9$ and $\kappa_{2}=2$.

should be compared with other possible topologies of phase diagrams (for example, those of three-dimensional mixtures of particles of similar lengths), where the firstorder I-N transition has two binodals that meet at the one-component limits of the phase diagram at finite pressure. This criterion is the one we have used to consider the transitions found here as I-N demixing transitions instead of the standard I-N orientational transition.

As can be seen from Fig. 5. at higher pressure from 
the tricritical point there is a triple intersection between two first order transition lines (the I binodal of the $\mathrm{I}_{-} \mathrm{N}_{1}$ transition and the $\mathrm{N}_{2}$ binodal of the $\mathrm{N}_{1}-\mathrm{N}_{2}$ transition) and the second order I- $\mathrm{N}_{2}$ transition line. This point is called in the literature a critical endpoint. An important remark to make is that this critical endpoint located at $x_{1}^{*}=0.0144$ and $\beta P^{*} v_{1}=16.2775$ does not exactly coincide with the predicted result from the bifurcation analysis. If we look at the lower intersection between the curve corresponding to $\kappa_{2}=2$ and the vertical line located at $\lambda \approx 0.222$ in Fig. [4 we find the value $x_{1}^{*}$ which corresponds to $\beta P v_{1}=13.8014$, which is lower than the above mentioned critical endpoint pressure. The reason for this disagreement can be elucidated if we plot the Gibbs free energy per particle of the mixture as a function of $x_{1}$ for three different values of the pressure, $\beta P v_{1}=13.8014$, which is the pressure corresponding to the upper tricritical point as obtained from the bifurcation analysis [the open circle in the inset of Fig. 6(a) corresponds to the bifurcation point]; $\beta P v_{1}=16.2775$, which corresponds to the triple intersection [Fig. [6(b)] where the bifurcation and the coexistence points coalesce; and $\beta P v_{1}=18.7536$, where the two coexistence points already lie on the nematic branch.

The main conclusion we can draw from this is that the bifurcation analysis predicts exactly the tricritical point (which corresponds to the lower pressure or higher $x_{1}^{*}$ in Fig. 4) and gives an approximate value for the critical endpoint.

In the light of these results we can interpret Fig. 31(a) and try to predict, as an example, the demixing behavior of a mixture of freely rotating HR with $\kappa_{2}=1$ and $\kappa_{1}=10^{2}$. If we increase the pressure $\beta P v_{1}\left(x_{1}^{*}\right)$ following the I- $\mathrm{N}_{u}$ spinodal curve we find that at a composition defined as the intersection with the solid line (the tricritical point) the mixture begins to demix into I and $\mathrm{N}_{u}$ phases. The demixing gap becomes wider with pressure, and at high pressures the lower intersection with the dashed line defines the approximate location of a critical endpoint where the first order I- $\mathrm{N}_{u}$ and $\mathrm{N}_{u}-\mathrm{N}_{t}$ transitions coalesce with a second order I- $\mathrm{N}_{t}$ transition. Increasing further the pressure we should have a $\mathrm{N}_{u}-\mathrm{N}_{t}$ coexistence and, ultimately, a coexistence between two uniaxial nematic phases. This latter coexistence could be predicted if we take into account the phase diagram for the onecomponent system, where the $\mathrm{N}_{t}$ phase is sandwiched between the I and $\mathrm{N}_{u}$ phases [33].

\section{PHASE DIAGRAMS OF HDR MIXTURES}

In this section we want to explicity discuss the demixing scenarios that may occur in binary mixtures of hard particles. We will focus our attention on HDR mixtures. For this purpose we have parameterized the orientational distribution functions of each species as

$$
h_{\mu}(\phi)=\frac{1}{\pi I_{0}\left(\alpha_{\mu}\right)} \exp \left(\alpha_{\mu} \cos 2 \phi\right)
$$
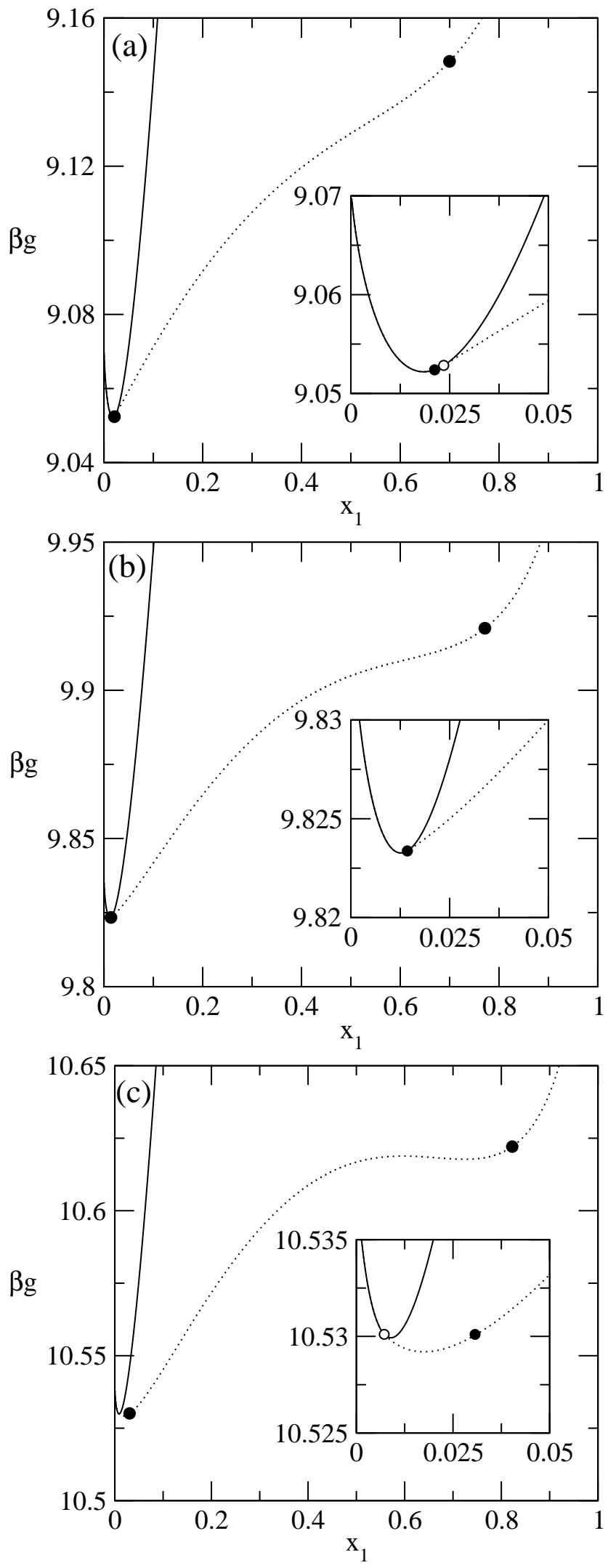

FIG. 6: Gibbs free energy per particle in reduced units $\beta g$ as a function of $x_{1}$ for the Zwanzig mixture with species having $\kappa_{1}=9$ and $\kappa_{2}=2$. The values of the pressure are $\beta P v_{1}=13.8014(\mathrm{a}), \beta P v_{1}=16.2775(\mathrm{~b})$, and $\beta P v_{1}=18.7536$ (c). The solid and dotted lines correspond to the I and $\mathrm{N}$ branches, respectively. The filled circles represent the coexistence points. The insets are enlargements of the neighborhood of the bifurcation points, which are shown by open circles. Panel (a) shows the loss of convexity of $\beta g$ in the $\mathrm{N}$ brach at the bifurcation point. 

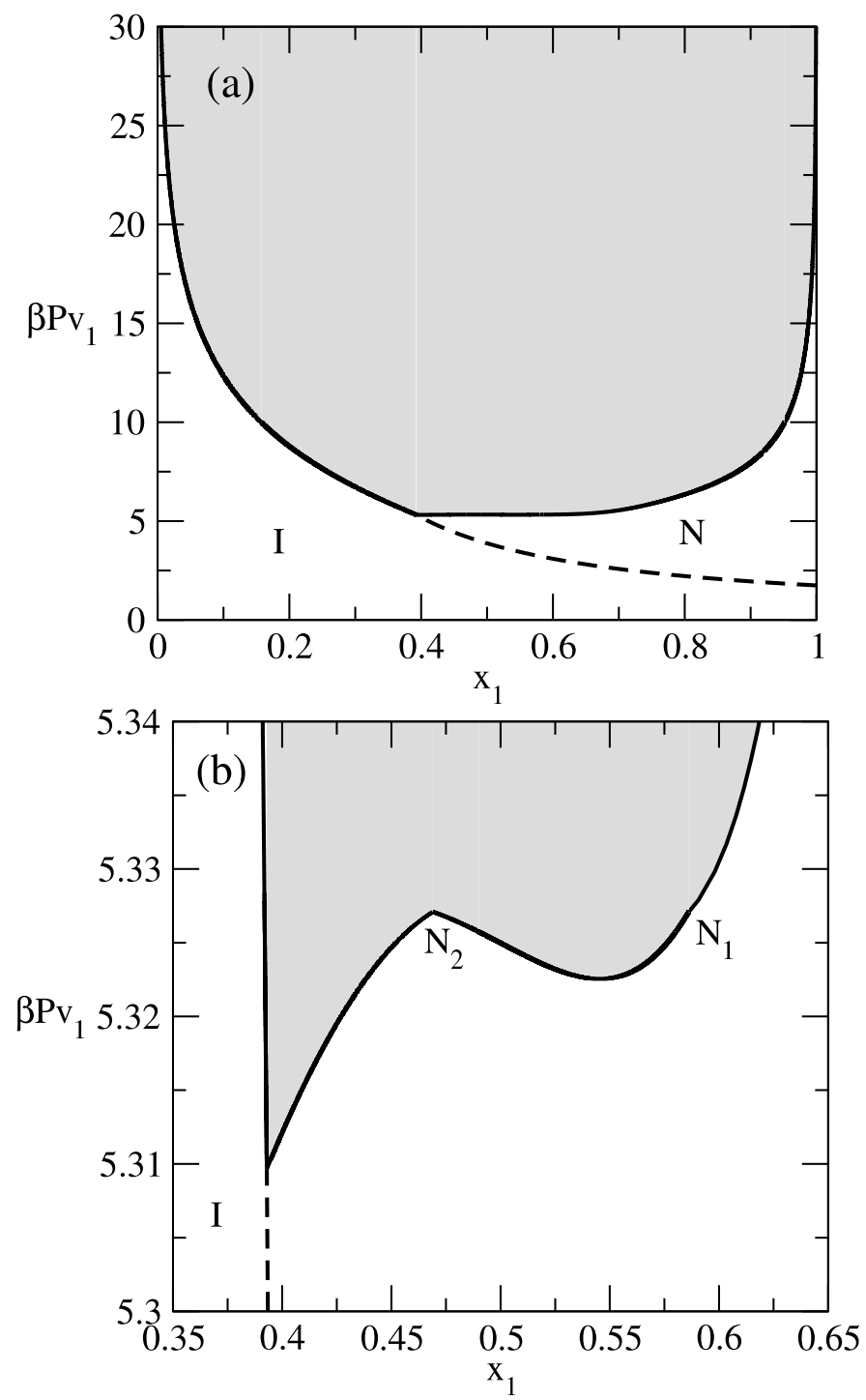

FIG. 7: (a) Phase diagram of the mixture of hard disks $\kappa_{2}=$ 1 and HDR with $\kappa_{1}=10$. (b) A zoom taken around the tricritical point.

where $I_{0}(x)$ is the zeroth-order modified Bessel function. This parametrization fulfills the normalization constraint $\int_{0}^{\pi} d \phi h_{\mu}(\phi)=1$. The free energy per volume (see Sec. II for its expression) was minimized with respect to $\alpha_{\mu}$ $(\mu=1,2)$ and the double-tangent construction on the thermodynamic potential $\beta g\left(x_{1}\right)$ (the Gibbs energy per particle in reduced units as a function of the composition of the mixture for a fixed pressure) was used to calculate the coexistence values of the composition of the mixture and the packing fraction.

The results for a mixture of hard disks $\left(\kappa_{2}=1\right)$ and HDR with $\kappa_{1}=10$ are shown in Figs. Z7(a) and 7 (b). For low pressures there is a continuous I-N transition at a pressure which agrees with that calculated from the spinodal packing fraction curve (34). At some composition $\left(x_{1}^{*}=0.393\right)$ we find a tricritical point from which the

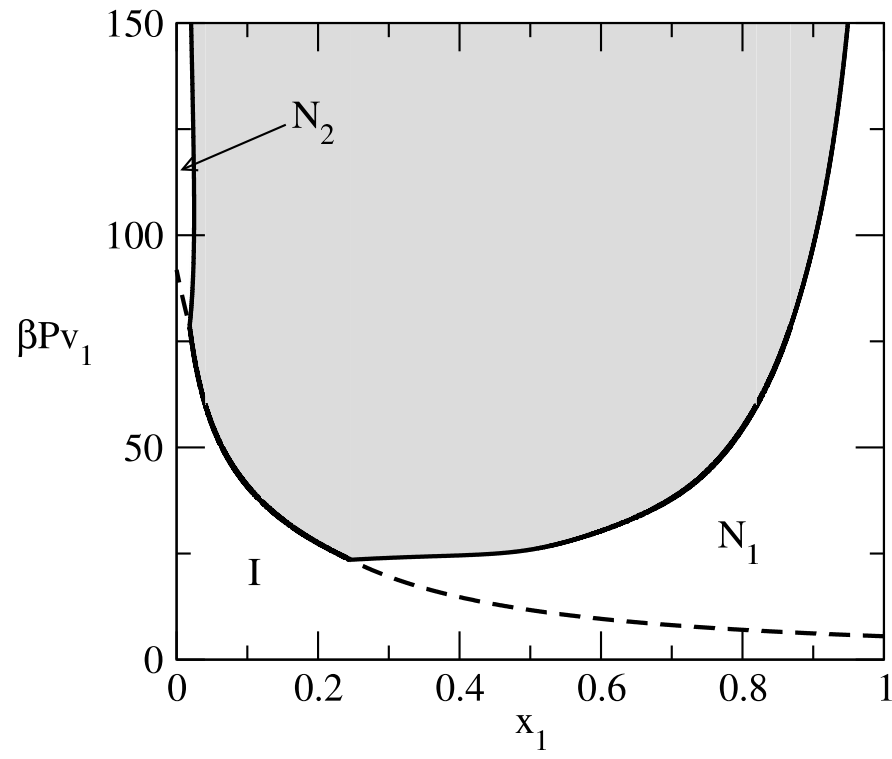

FIG. 8: Phase diagram for a HDR mixture with $\kappa_{1}=5$ and $\kappa_{2}=2$.

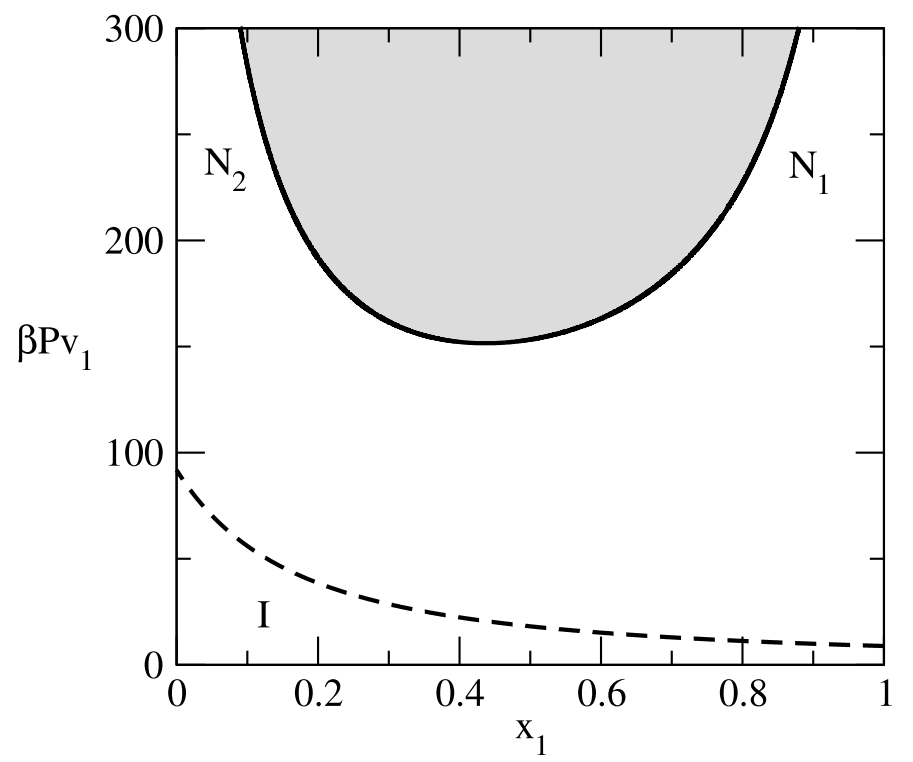

FIG. 9: Phase diagram for a HDR mixture with $\kappa_{1}=4$ and $\kappa_{2}=2$.

mixture begins to demix into the I phase (rich in discs) and the $\mathrm{N}$ phase (rich in rods). The location of the tricritical point should be compared with that resulting from the bifurcation analysis $\left(x_{1}^{*}=0.434\right)$ which gives the exact result. The difference is due to the parametrization used. As we have already pointed out the value of $x_{1}^{*}$ depends on the coefficient $B^{*}$ which in turn depends on the $a_{\mu \nu}^{(k)}(k=1, \ldots, 4)$. These are the expansion coefficients of the free-energy difference around the bifurcation point up to fourth order. Although the used parametrization captures the right second order terms, further terms are only approximate due to the restriction on the minimiza- 
tion variables to be only one per species.

An interesting result is shown in Fig. 7(b), a zoom of Fig. 7(a) around the tricritical point. As we can see this part of the phase diagram exhibits a $\mathrm{N}_{1}-\mathrm{N}_{2}$ coexistence which ends in a critical point, along with the presence of a $\mathrm{I}-\mathrm{N}_{1}-\mathrm{N}_{2}$ triple point. As these features are similar to those observed in the Zwanzig mixture (not shown here), where no parametrization was used, we are confident that this scenario is qualitatively correct.

Two other phase diagrams are shown in Figs. 8 and 9 for different mixtures. The topology of the first one is similar to that found in the Zwanzig mixture, where the I- $\mathrm{N}_{1}$ demixing is followed by $\mathrm{N}_{1}-\mathrm{N}_{2}$ coexistence and the phase diagram includes one tricritical and one critical endpoint.

The third phase diagram (see Fig. 9) includes an I-N transition which is always of second order and, at high pressures, a $\mathrm{N}_{1}-\mathrm{N}_{2}$ demixing ending in a critical point. This kind of phase diagram is typical of mixtures of particles with similar aspect ratios. Since the pressures at which the demixing transition occurs are rather high, we expect this transition to be metastable with respect to phases with partial or full spatial order. Comparing the phase diagram of Fig. 9] with the exact bifurcation analysis results (see Fig. 11), we can conclude that, even though I-N demixing is already allowed from $\lambda \leq 0.595$, the parametrization used changes the demixing behavior of the mixture. An exact free-energy minimization for the same value of $\kappa_{2}$ and for $\lambda>0.595$ should qualitatively give a phase diagram similar to that shown in Fig. 9

\section{CONCLUSIONS}

While I-I demixing is forbidden in two-dimensional hard-body additive mixtures of anisotropic particles ac- cording to SPT [25], we have shown in the present work that the inclusion of ordered phases with orientational symmetry breaking changes completely the demixing scenario. The phase diagrams characteristic of these mixtures can exhibit I-N and N-N demixing. We have used a bifurcation analysis to demonstrate rigorously that I-N demixing occurs. On the other hand, explicit calculations of the phase diagrams of HDR, using an accurate parametrization, have been performed in order to show the occurrence of $\mathrm{N}-\mathrm{N}$ demixing. The simple structure of SPT allowed us to obtain analytically the stability criterion for the mixture as a function of packing fraction, composition and shape of the constituent particles. Thus, using this procedure, we can predict the I-N demixing scenarios for mixtures of HDR and HR.

To show the relative stability of these demixed phases with respect to nonuniform phases (e.g., solid phases), we would need to carry out a full minimization, with respect to the density profile $\rho_{\mu}(\mathbf{r}, \phi)$ (which also depends on spatial variables), of a density functional constructed in such a way that it recovers the SPT in the uniform limit. This work is a task in progress.

Finally, we expect that all the demixing scenarios predicted here be confirmed by computer simulations of hard-body mixtures in two dimensions. These simulations are still lacking.

\section{ACKNOWLEDGMENTS}

One of the authors (Y. M.-R.) was supported by a Ramón y Cajal research contract from Ministerio de Educación y Ciencia (Spain). This work is part of research projects Nos. BFM2003-0180, BFM2001-0224-C02-01, BFM2001-0224-C02-02, and BFM2001-1679-C03-02 of the Ministerio de Educación y Ciencia (Spain).
[1] M. Dijkstra, R. van Roij, and R. Evans, Phys. Rev. Lett. 81, 2268 (1998); Phys. Rev. E 59, 5744 (1998).

[2] N. G. Almarza and E. Enciso, Phys. Rev. E 59, 4426 (1998).

[3] A. Buhot and W. Krauth, Phys. Rev. Lett. 80, 3787 (1997).

[4] Y. Martínez-Ratón and J. A. Cuesta, Phys. Rev. E 58, R4080 (1998).

[5] E. Velasco, G. Navascués, and L. Mederos, Phys. Rev. E 60, 3158 (1999).

[6] L. Lafuente and J. A. Cuesta, Phys. Rev. Lett. 89, 145701 (2002).

[7] T. Biben, P. Blandon, and D. Frenkel, J. Phys.: Condens. Matter 8, 10799 (1996).

[8] R. van Roij and B. Mulder, J. Phys. II 4, 1763 (1994).

[9] H. H. Wensink, G. J. Vroege, and H. N. W. Lekkerkerker, J. Chem. Phys. 115, 7319 (2001).

[10] S. Dubois and A. Perera, J. Chem. Phys. 116, 6354 (2002); A. Perera, K. Cassou, F. Ple, and S. Dubois, Mol.
Phys. 100, 3409 (2002); A. Perera, J. Mol. Liq. 109, 73 (2004).

[11] S. Varga, A. Galindo, and G. Jackson, J. Chem. Phys. 117, 7207 (2002); A. Galindo, A. J.Haslam, S. Varga, G. Jackson, A. G. Vanakaras, D. J. Photinos, and D. A. Dunmur, ibid. 119, 5216 (2003).

[12] Y. Martínez-Ratón and J. A. Cuesta, J. Chem. Phys. 118, 10164 (2003).

[13] M. Schmidt and A. R. Denton, Phys. Rev. E 65, 021508 (2002)

[14] S. M. Oversteegen and H. N. W. Lekkerkerker, J.Chem. Phys. 120, 2470 (2003).

[15] R. van Roij, and B. Mulder, Phys. Rev. E 54, 6430 (1996); R. van Roij, B. Mulder, and M. Dijkstra, Physica A 261, 374 (1998).

[16] M. Dijkstra and R. van Roij, Phys. Rev. E 56, 5594 (1997).

[17] S. Varga, A. Galindo, and G. Jackson, Mol. Phys. 101, 817 (2003). 
[18] K. R. Purdy, S. Varga, A. Galindo, G. Jackson, and S. Fraden, Phys. Rev. Lett. 94, 057801 (2005).

[19] H. Reiss, H. L. Frisch, and J. L. Lebowitz, J. Chem. Phys. 31, 369 (1959).

[20] M. A. Cotter and D. E. Martire, J. Chem. Phys. 52, 1902 (1970); 53, 4500 (1970); M. A. Cotter and D. C. Wacker, Phys. Rev. A 18, 2669 (1978).

[21] G. Lasher, J. Chem. Phys. 53, 4141 (1970).

[22] B. Barboy and W. Gelbart, J. Chem. Phys. 71, 3053 (1979).

[23] A. Isihara, J. Chem. Phys. 18, 1446 (1950).

[24] T. Kihara, Rev. Mod. Phys. 25, 831 (1953).

[25] J. Talbot, J. Chem. Phys. 106, 4696 (1997).

[26] Y. Rosenfeld, Phys. Rev. Lett. 63, 980 (1989).

[27] P. Tarazona, Phys. Rev. Lett. 84, 694 (2000).

[28] R. Tenne and E. Bergmann, Phys. Rev. A 17, 2036 (1978); R. J. Bearman and R. M. Mazo, J. Chem. Phys. 88, 1235 (1988); ibid. 91, 1227 (1989); R. Mazo and R. J. Bearman, ibid. 93, 6694 (1990).
[29] R. Mountain and A. H. Harvey, J. Chem. Phys. 94, 2238 (1991); F. Saija and P.V. Giaquinta, ibid. 117, 5780 (2002).

[30] R. F. Kayser and H. J. Raveché, Phys. Rev. A 17, 2067 (1978).

[31] P. van der Schoot, J. Chem. Phys. 106, 2355 (1997).

[32] M. A. Bates and D. Frenkel, J. Chem. Phys. 112, 10034 (2000).

[33] Y.Martínez-Ratón, E.Velasco, and L. Mederos, J. Chem. Phys. 122, 064903 (2005).

[34] H. Schlacken, H. -J. Mogel, and P. Schiller, Mol. Phys. 93, 777 (1998).

[35] A. Poniewierski and T. J. Sluckin, Phys. Rev. A 43, 6837 (1991).

[36] Note that in the one-component limit the condition $H^{*}=$ 0 should be substituted by $\left(\varkappa_{T}^{-1}\right)^{*}=0$ [where $\varkappa_{T}^{-1}=$ $\rho(\partial \beta P / \partial \rho)_{T}$ is the inverse isothermal compressibility]. 